\title{
COMPARISON OF THE ACTIVITIES OF THE DEEP TRUNK MUSCLES MEASURED USING INTRAMUSCULAR AND SURFACE ELECTROMYOGRAPHY
}

\author{
YU OKUBO \\ Graduate School of Comprehensive Human Science, University of Tsukuba \\ 1-1-1 Tennodai, Tsukuba, Ibaraki, 305-8574, Japan \\ y-okubo@tius-hs.jp \\ KOJI KANEOKA \\ Faculty of Sports Science, Waseda University \\ 2-579-15, Mikajima, Tokorozawa, Saitama 359-1192, Japan \\ kaneoka@waseda.jp \\ ATSUSHI IMAI \\ Graduate School of Comprehensive Human Science, University of Tsukuba \\ 1-1-1 Tennodai, Tsukuba, Ibaraki, 305-8574, Japan \\ ITSUO SHIINA \\ Graduate School of Comprehensive Human Science, University of Tsukuba \\ 1-1-1 Tennodai, Tsukuba, Ibaraki, 305-8574, Japan \\ MASAKI TATSUMURA \\ Graduate School of Comprehensive Human Science, University of Tsukuba \\ 1-1-1 Tennodai, Tsukuba, Ibaraki, 305-8574, Japan \\ SHIGEKI IZUMI \\ Faculty of Sports and Health Studies, Hosei University \\ 4342 Aiharacho, Machida, Tokyo, 194-0298, Japan \\ SHUMPEI MIYAKAWA \\ Graduate School of Comprehensive Human Science, University of Tsukuba \\ 1-1-1 Tennodai, Tsukuba, Ibaraki, 305-8574, Japan
}

\begin{abstract}
Surface electromyography (EMG) has been used to estimate deep trunk muscle activity. However, it remains unknown whether surface EMG provides an accurate estimation of this activity. The purposes of this study were to compare surface and intramuscular EMG activity measurements and investigate the efficacy of surface EMG measurement for the transversus abdominis (TrA) and the multifidus (MF) muscles. Eight healthy men participated in the study. TrA and MF activities were simultaneously measured by both intramuscular and surface EMG during isometric trunk exercises. Spearman correlation coefficients for the relationship between the two activity measurements for the right TrA, left TrA, right MF and left MF were 0.55, 0.36, $0.67,0.79$, respectively. For the TrA, Bland-Altman plots revealed that mean differences between measurements obtained by intramuscular EMG and surface EMG were not close to zero, with a systematic bias toward higher surface EMG values. In conclusion, surface and intramuscular EMG activity measurements were strongly correlated for MF muscles, but poorly correlated for TrA muscles.
\end{abstract}

Keywords: Deep trunk muscles; electromyography; validation

Running heads: Validity of Surface Electromyography for Deep Trunk Muscles 


\section{Introduction}

Electromyography (EMG) is used to record muscle activity. There are two types of electromyographic (EMG) electrodes: intramuscular and surface electrodes. Intramuscular electrodes are typically fine-wire or needle electrodes; these are inserted into the muscle such that signals may be recorded directly. Although this method is invasive, it can measure the potential of a specific deep muscle and is minimally affected by crosstalk ${ }^{1}$. In contrast, surface EMG electrodes are attached to the skin overlying the muscle, and thus measure its potential indirectly. Surface electrodes simultaneously record potentials from several muscles (crosstalk), and therefore are not considered ideal for recording activity of deep muscles.

In clinical practice, lumbar stabilization exercises are commonly recommended for rehabilitation of the lumbar spine $e^{2}$. It has been reported that muscle control the stability of the lumbar spine within a neutral $z^{2} e^{3}$, and that an important contribution is made by co-contraction of the trunk muscles, including deep muscles such as the transversus abdominis $(\operatorname{Tr} A)$ and the multifidus $(M F)^{4-6}$. Therefore, lumbar stabilization exercises should be performed to facilitate contraction of the deep trunk muscles.

Surface electrodes have been used to assess activity of the deep trunk muscles in some studies ${ }^{7-9}$. It is therefore essential to determine whether surface EMG measurement provide a valid estimate of the independent activities of the TrA and MF. Arokoski et al ${ }^{10}$ documented a correlation between normalized intramuscular and surface EMG of MF activities. However, in contrast, Stokes et $\mathrm{al}^{11}$ found that accurate measurement of MF required the use of intramuscular EMG. In the case of $\operatorname{TrA}$, few reports have compared EMG amplitudes between intramuscular and surface EMG methods. Therefore, the purpose of this study was to examine the validity of surface EMG measurement of TrA and MF activity by directly comparing the surface EMG with simultaneous intramuscular EMG recording for these two muscles.

\section{Methods}

\subsection{Subjects}

Participants included 8 healthy men (age, 24.0 [0.8] years (mean [SD]); height, 170.8 [5.1] cm; body mass: $62.6[5.9] \mathrm{kg}$ ) who did not have low back pain at the time of the experiment. Those with a history of lumbar spine disorder, neurological disorder, or surgery of the spine were excluded from the study. The participants were informed about the purpose and protocol of the study before they were asked to sign an informed consent form. The study was approved by the Ethics Committee of the University of Tsukuba (approval number, 590).

\subsection{Intramuscular EMG}

Fine-wire bipolar electrodes for intramuscular EMG were fabricated from two strands of urethane-coated stainless-steel wire (diameter $0.05 \mathrm{~mm}$; UNIQUE MEDICAL Co. Ltd., Japan) threaded into 23-gauge hypodermic needles after removing $2 \mathrm{~mm}$ of urethane and bending back the tips to form 1 and $2 \mathrm{~mm}$ hooks. The electrodes were sterilized in an autoclave (HighClave HVE-50: Hirayama Manufacturing Corp., Japan) at $121^{\circ} \mathrm{C}$ for 20 minutes. The electrodes were inserted bilaterally into the lower region of the TrA (adjacent to the anterior superior iliac spine (ASIS)) ${ }^{12}$ and MF muscles (approximately $2 \mathrm{~cm}$ lateral to the L5 spinous process) ${ }^{10}$ under ultrasonic guidance by an orthopedic surgeon (Fig. 1). When the target muscle was reached, the needle was withdrawn, leaving the fine-wire electrode within the muscle. The muscle was then stimulated with an electric stimulation device, and muscular contraction was confirmed by ultrasound imaging.

\subsection{Surface EMG}

Before surface electrode attachment, the skin was rubbed with an abrasive and alcohol in order to reduce the skin impedance below $2 \mathrm{k} \Omega$. Pairs of disposable $\mathrm{Ag} / \mathrm{AgCl}$ surface electrodes (Vitrode F-150S; Nihon Kohden Co. Ltd., Japan) were attached to the skin overlying the bilateral $\operatorname{Tr} A$ and MF, and in parallel to the muscle fibers, with a center-to-center distance of $2 \mathrm{~cm}$. TrA electrodes were placed approximately 2 $\mathrm{cm}$ medial and inferior to the ASIS the site demonstrated by McGill et $\mathrm{al}^{13}$ to be optimal for measuring TrA activity. MF electrodes were placed $2 \mathrm{~cm}$ lateral to the L5 spinal process, a site used in several studies for surface electrode measurement of MF activity ${ }^{2,4,14}$. A reference electrode was placed over the sternum. 


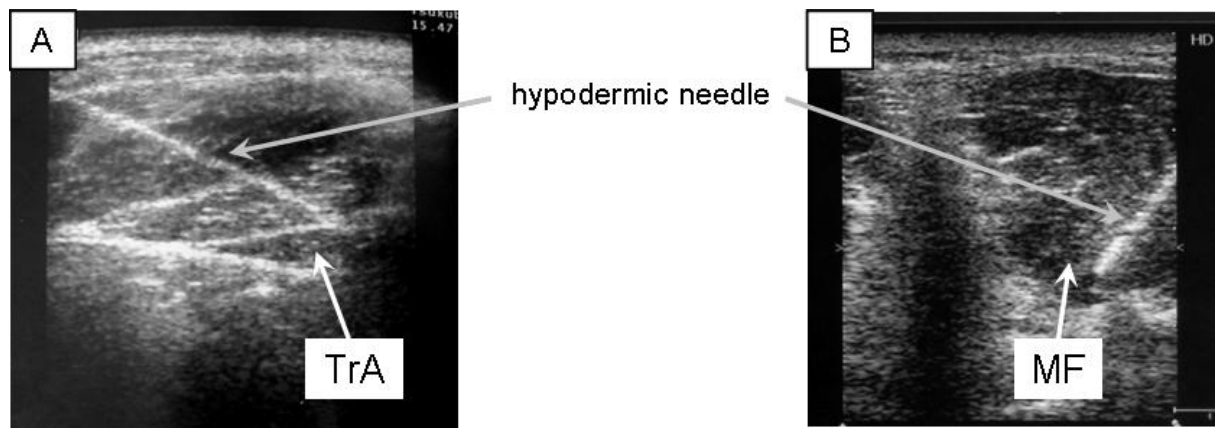

Fig. 1. Ultrasound imaging of hypodermic needle implantation of electrodes into the $\operatorname{Tr} A(A)$ and the MF (B)

\subsection{Procedure}

We chose high-load lumbar stabilization exercises as experimental tasks. Subjects were instructed to perform the following five isometric exercises: Elbow-Toe with right arm and left leg lift, Hand-Knee with right arm and left leg lift, Back Bridge with left leg lift, Side Bridge with left leg lift and Curl Up (Fig. 2). Each exercise was performed once, while EMG signals were recorded. Subjects were instructed to maintain the respective position for 3 seconds in each exercise, and were allowed to rest between the exercises. Data were recorded for all eight participants during one successful attempt of each of the five exercises to give a total of $40 \mathrm{EMG}$ values. These data were used for further analyses.

\subsection{EMG Data Analysis}

All EMG signals were amplified 1,000 fold using an amplifier (MEG-6116, JB-640J NIHON KOHDEN Co., Ltd., Japan). The sampling frequency was $1,000 \mathrm{~Hz}^{15}$. The raw data were band-pass filtered between 20 to $500 \mathrm{~Hz}$ and full-wave rectified using analysis software (BIMUTAS-Video: Kissei Comtec Co. Ltd., Japan).

For each exercise, a root-mean-square (RMS) was calculated for a 1-second interval when the posture was static. The RMS calculated during each exercise was normalized as a percentage of the RMS during a maximum voluntary contraction task (\%MVC). For the TrA, the MVC task was the maximal expiratory maneuver with abdominal hollowing performed in the sitting position ${ }^{8,16}$. For the MF, the MVC task was trunk extension performed in the prone position with application of manual resistance to the upper thoracic area and without leg movement ${ }^{17,18}$. Manual resistance was gradually increased applied up to the subject's limit, then held for 3 seconds. RMS for the MVC tasks was calculated for a 1-second interval when posture was stable, including the peak amplitude.
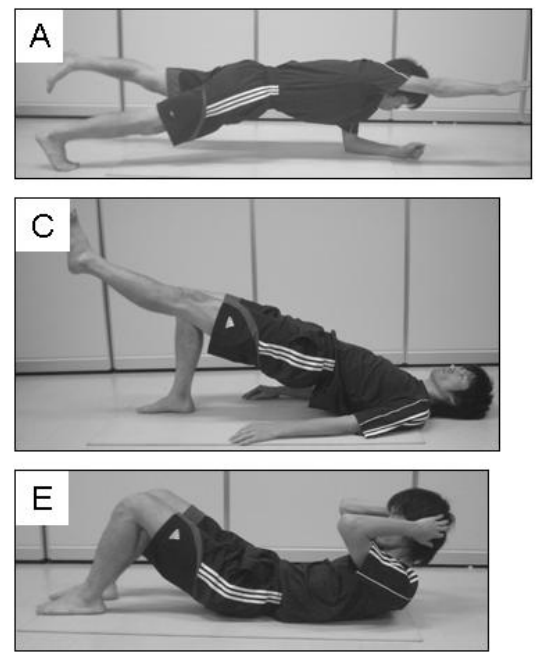
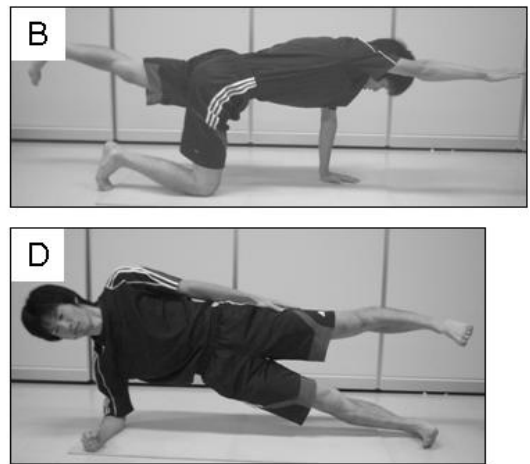

Fig. 2. Illustrations of the exercises performed by the study participants
(A) Elbow-Toe with right arm and left leg lift,
(B) Hand-Knee with right arm and left leg lift (C) Back Bridge with left leg lift,

(D) Side Bridge with left leg lift, (E) Curl Up 


\subsection{Statistical Analysis}

Smirnov-Grubbs rejection test was used to exclude outlier data for each muscle. Spearman's rank correlation coefficient was calculated to assess the relationship between intramuscular and surface EMG activity measurement for each muscle. Measurements obtained using two EMG techniques were also compared using the Bland-Altman plot, which assesses agreement between two assays. This statistical approach is more appropriate for this purpose than $t$ tests or intraclass correlation coefficients, and has been applied in several muscle biology studies to compare different measurement techniques ${ }^{21,22}$.

JMP 6.0 for Windows (SAS Institute Japan Co. Ltd., Japan) was used for statistical analysis.

\section{Results}

On the basis of the findings of the Smirnov-Grubbs rejection test, we excluded two outlying values for the right TrA, and one value each for the left TrA, right MF and left MF.

Intramuscular and surface EMG measurements of muscle activities, as \%MVC, were then compared for the right TrA, left TrA, right MF, and left MF (Fig. 3-6). The Spearman's rank correlation coefficients for the four muscles were 0.55, 0.36, 0.67 and 0.79, respectively (panel A in Fig. 3-6).

Bland-Altman plots of the data for each muscle are also shown (panel B in Fig. 3-6). For TrA, the fan-shaped Bland-Altman plots show a systematic bias that the difference between the two measurements tended to increase as the mean values increased. For the right and left $\operatorname{Tr} \mathrm{A}$, the mean differences between the two measurements were 8.5\% MVC and 10.4\% MVC, respectively, demonstrating higher surface EMG values than the corresponding intramuscular EMG values (Fig. 3B and 4B). On the other hands, for the right and left MF the mean difference were near zero (right MF, $0.94 \% \mathrm{MVC}$; left MF, $2.46 \% \mathrm{MVC}$ ) (Fig. 5B and 6B). The limits of agreement (mean difference $\pm 2 \mathrm{SD}$ ) for the right TrA and left TrA were $-58.6 \% \mathrm{MVC}$ to $67.1 \% \mathrm{MVC}$ and $-16.7 \% \mathrm{MVC}$ to $37.4 \% \mathrm{MVC}$, respectively. The range was narrow for the limits of agreement for the left MF (-14.5\%MVC to $19.4 \% \mathrm{MVC})$ than that of the TrA.

\section{Discussion}

This study was designed to evaluate the validity of using surface electrodes to estimate activity of the TrA and MF muscles by simultaneously recording with intramuscular and surface electrodes.

For the TrA, the Spearman correlation coefficients between the two measurements were low (right TrA, 0.55: left TrA, 0.36). Bland-Altman plots of the differences in the two measurements revealed that surface EMG produced higher measurements of TrA activity than did intramuscular EMG, and the limits of agreement were broader. These results indicate that the surface electrodes did not measure independent TrA activity, but the combined activities of the $\operatorname{TrA}$ with other muscles, most likely internal oblique muscle (IO). Indeed, McGill et $\mathrm{al}^{13}$ have reported that $\operatorname{TrA}$ activity recorded using intramuscular
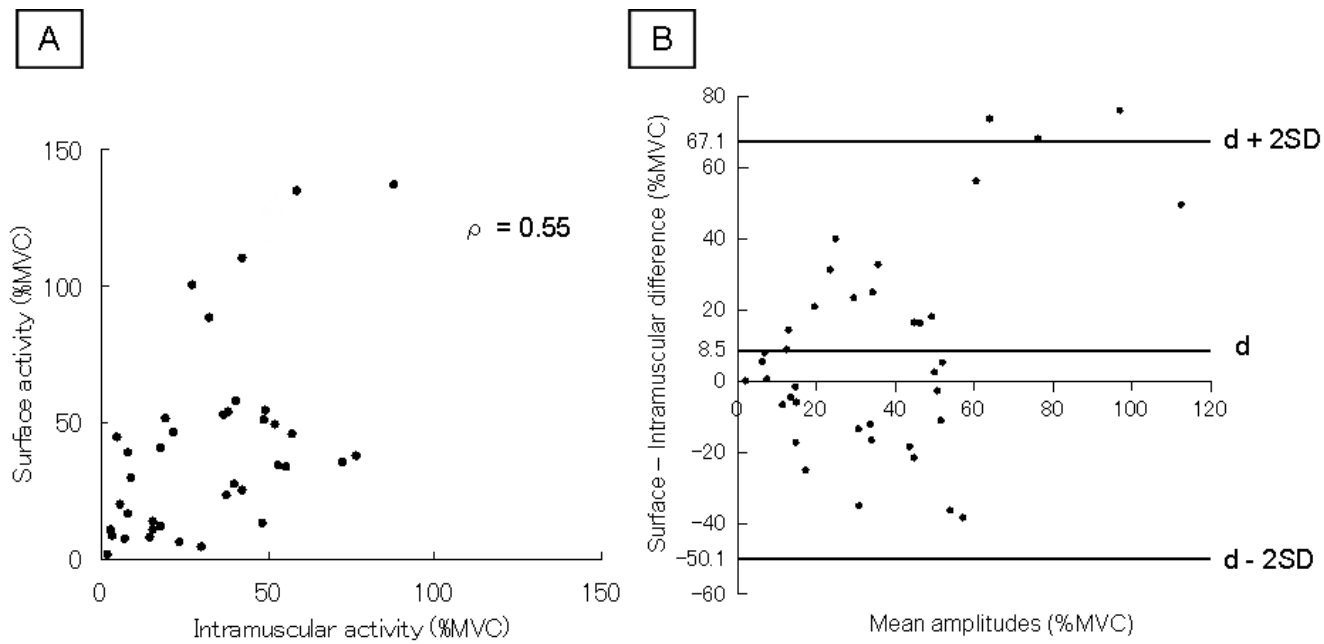

Fig. 3. Correlation between surface and intramuscular activity measurements (A) and Bland-Altman plot (B) for the right TrA. $\mathrm{d}=$ mean difference, $\mathrm{SD}=$ standard deviation, $\rho=$ Spearman's rank correlation coefficient 


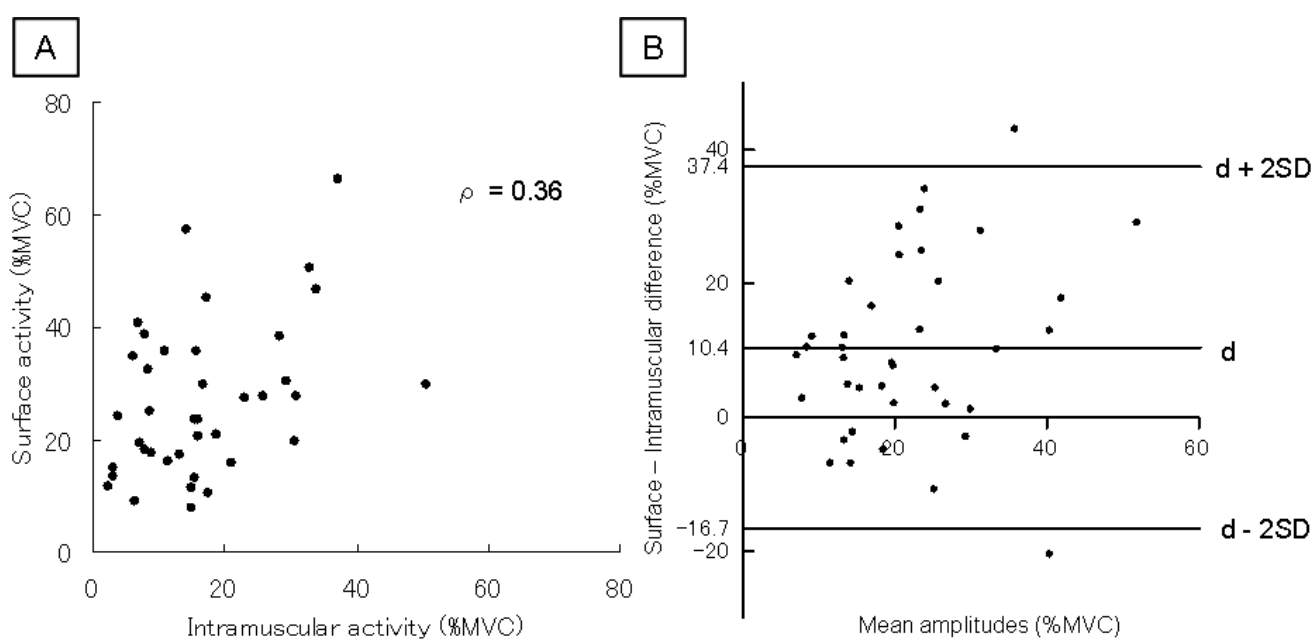

Fig. 4. Correlation between surface and intramuscular activity measurements (A) and Bland-Altman plot (B) for the left $\operatorname{TrA}$ $\mathrm{d}=$ mean difference, $\mathrm{SD}=$ standard deviation, $\rho=$ Spearman's rank correlation coefficient
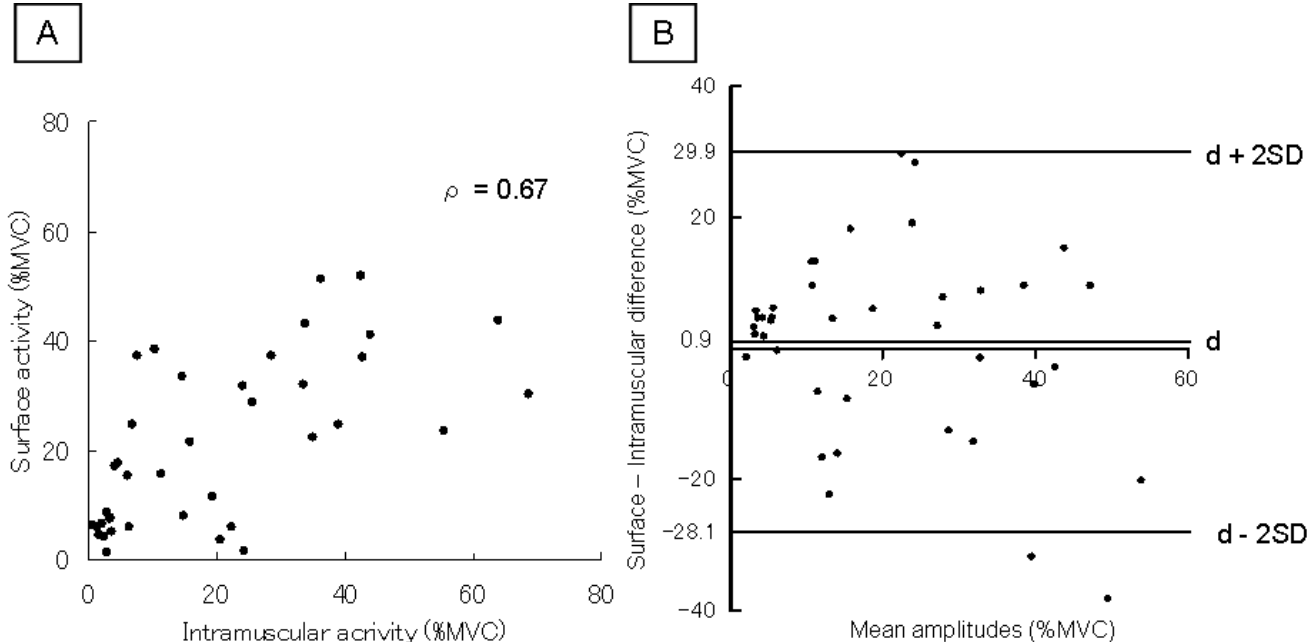

Fig. 5. Correlation between surface and intramuscular activity measurements (A) and Bland-Altman plot (B) for of the right MF. $\mathrm{d}=$ mean difference, $\mathrm{SD}=$ standard deviation,$\rho=$ Spearman's rank correlation coefficient
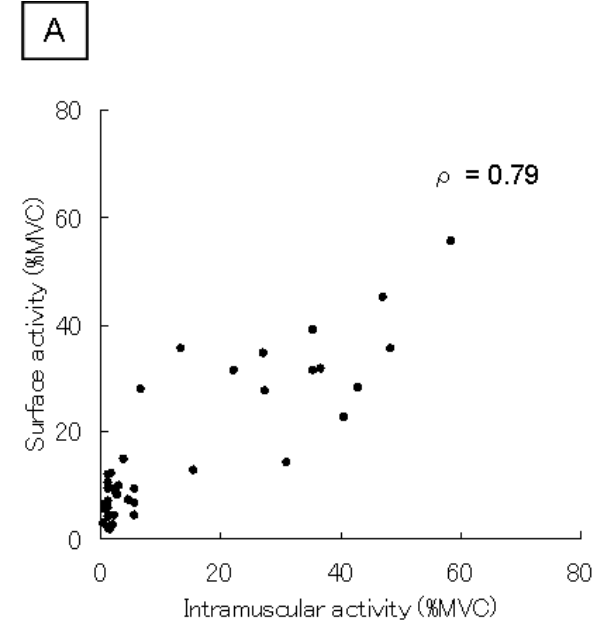

\section{B}

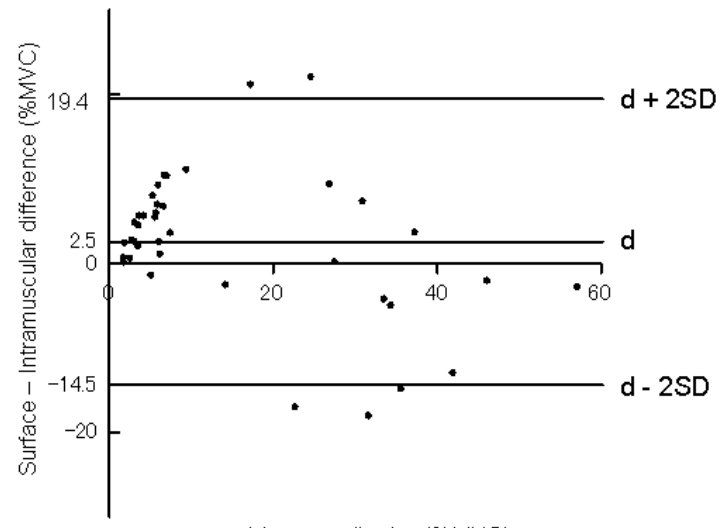

Mean amplitudes (\%MVC)

Fig. 6. Correlation between surface and intramuscular activity measurements (A) and Bland-Altman plot (B) for the left MF. $\mathrm{d}=$ mean difference, $\mathrm{SD}=$ standard deviation, $\rho=$ Spearman's rank correlation coefficient 
electrodes correlated well with the IO activity. In that study, the surface electrodes used to measure TrA activity were positioned approximately $2 \mathrm{~cm}$ medial and inferior to the ASIS, overlapping the site used to measure the IO activity, but not, however, the external oblique muscle ${ }^{23}$. Additionally, Brown et $\mathrm{al}^{24}$ have indicated the presence of shear connections by the muscular layer situated between the $\operatorname{Tr} \mathrm{A}$ and IO. Therefore, it may be difficult to estimate the independent activity of $\operatorname{TrA}$ using surface electrodes. Marshall et $\mathrm{al}^{25}$ also noted that although surface EMG measurement could identify the onset of deep abdominal muscles, it likely represented combined $\operatorname{TrA} / \mathrm{IO}$ activity and not independent $\operatorname{Tr} \mathrm{A}$ activity. Some of the values we recorded for TrA exceeded $100 \%$ MVC. This may be due to the way that EMG activity was normalized. An MVC method for TrA has not established, and we adopted the same task used in previous studies ${ }^{8,16}$ to estimate this MVC. Therefore, $\operatorname{TrA}$ activity might have been overestimated due to underestimation of the MVC.

With respect to MF activity, Spearman correlation coefficients between the two measurements were high (right MF, 0.67: left MF, 0.79), and Bland-Altman plots showed no evidence of systematic or proportional biases. For the MF, therefore, surface EMG measurement at the L5 vertebral level may be satisfactory. Consistent with our finding, Arokoski et a ${ }^{10}$ suggested that surface EMG measurement can be used to estimate MF activity during various therapeutic exercises. On the other hand, Stokes et $\mathrm{al}^{11}$ reported that MF activity measured using surface electrodes at the L2 and L4 vertebral levels correlated more strongly with longissimus (LG) activity than with MF activity measured using intramuscular electrodes. The difference between that outcome and our present finding is likely attributable to the difference in MF measurement site. The MF has separate bands from each spinous process of the lumbar spine $^{26}$, and activity may differ at each vertebral level. Since many researchers ${ }^{8,17,27,28}$ have used surface EMG electrodes at the L5 vertebral level, we measured the MF activity at this site. A large number of low \%MVC values were recorded for MF activity during exercises, and this circumstance may have strengthened the correlation between the two different measurements for MF activity. This point could be clarified in future work using exercises that produce higher MF activation.

We found bilateral differences in results for the TrA and MF, which is not unexpected with the asymmetric exercises performed in the study. The asymmetric muscle activity might be responsible for the right/left differences in correlation coefficients and limits of agreement for the TrA and MF.

Intramuscular EMG signal are highly localized, and in the present study, they were recorded at a signal site. Thus, a more extensive study could include data collection at multiple intramuscular sites. Moreover, inclusion of activity of adjacent muscles such as the IO and LG would elucidate the occurrence of crosstalk.

\section{Conclusion}

We compared surface EMG with the intramuscular EMG activity measurements for the tranversus abdominis and multifidus muscles. While there was high correlation between the two measurements techniques for the MF, in the case of the TrA the correlation was low, suggesting possible crosstalk in the surface EMG by adjacent muscles.

\section{Acknowledgement}

This study was supported by a Grant-in-Aid for Scientific Research (B) from the Japan Society for the Promotion of Science (grant number, 19300220). We would like to acknowledge the contribution of Fuminari Kaneko in providing guidelines on the use of intramuscular electrodes.

\section{References}

1. Solomonow M, Barratta R, Bernard M, Zhou B, Lu Y, Zhu M, Acheron S, Surface and wire EMG crosstalk in neighboring muscles, J Electromyogr Kinesiol 4: 131-142, 1994.

2. Kavcic N, Grenier S, McGill SM, Quantifying tissue loads and spine stability while performing commonly prescribed low back stabilization exercises, Spine 29: 2319-2329, 2004.

3. Panjabi MM, The stabilizing system of the spine Part 2: Neutral zone and stability hypothesis, J Spinal Disord 5:390-397, 1992.

4. Cholewicki J, McGill SM, Mechanical stability of the in vivo lumbar spine. Implications for injury and low back pain, Clin Biomech 11:1-15, 1996.

5. Cholewicki J, VanVliet JJ 4th, Relative contribution of trunk muscles to the stability of the lumbar spine during isometric exertions, Clin Biomech 17:99-105, 2002.

6. Stanton T, Kawchuk G., The effect of abdominal stabilization contractions on posteroanterior spinal stiffness, 
Spine 33:694-701, 2008.

7. Danneels LA, Coorevits PL, Cools AM, Vanderstraeten GG, Cambier DC, Witvrouw EE, De CH, 2002. Differences in electromyographic activity in the multifidus muscle and the iliocostalis lumborum between healthy subjects and patients with sub-acute and chronic low back pain, Eur Spine J 11:13-19, 2002.

8. Marshall PW, Murphy BA, Core stability exercises on and off a Swiss ball, Arch Phys Med Rehabil 86:242-249, 2005.

9. Marshall PW, Murphy BA, Changes in muscle activity and perceived exertion during exercises performed on a swiss ball, Appl Physiol Nutr Metab 31:376-383, 2006.

10. Arokoski JP, Kankaanpaa M, Valta T, Juvonen I, Partanen J, Taimela S, Lindgren KA, Airaksinen O, Back and hip extensor muscle function during therapeutic exercises, Arch Phys Med Rehabil 80:842-850, 1999.

11. Stokes IA, Henry SM, Single RM, Surface EMG electrodes do not accurately record from lumbar multifidus muscles, Clin Biomech 18:9-13, 2003.

12. Urquhart DM, Hodges PW, Story IH, Postural activity of the abdominal muscles varies between regions of these muscles and between body positions, Gait Posture 22:295-301, 2005.

13. McGill S, Juker D, Kopf R, Appropriately placed surface EMG electrodes reflect deep muscle activity (psoas, quadratus lumborum, abdominal wall in the lumbar spine) in the lumbar spine, J Biomech 29:1503-1507, 1996.

14. Stevens VK, Bouche KG, Mahieu NN, Coorevits PL, Vanderstraeten GG., Danneels LA, Trunk muscle activity in healthy subjects during bridging stabilization exercises, BMC Musculoskelet Disord 7:75, 2006.

15. Andersson EA, Grundstrom H, Thorstensson A, Diverging intramuscular activity patterns in back and abdominal muscles during trunk rotation, Spine 27:E152-160, 2002.

16. Ferreira PH, Ferreira ML, Hodges PW, Changes in recruitment of the abdominal muscles in people with low back pain; ultrasound measurement of muscle activity, Spine 29:2560-2566, 2004.

17. Ekstrom RA, Donatelli RA, Carp KC, Electromyographic analysis of core trunk, hip, and thigh muscles during 9 rehabilitation exercises, J Orthop Sports Phys Ther 37:754-762, 2007.

18. Stevens VK, Vleeming A, Bouche KG, Mahieu NN, Vanderstraeten GG, Danneels LA, Electromyographic activity of trunk and hip muscles during stabilization exercises in four-point kneeling in healthy volunteers, Eur Spine J 16:711-718, 2007.

19. Altman DG, Bland JM, Measurement in medicine: the analysis of method comparison studies, The Statistician 32:307-317, 1983.

20. Bland JM, Altman DG, Statistical methods for assessing agreement between two methods of clinical measurement, The Lancet 8:307-310, 1986.

21. Mannion A, Troke M, A comparison of two motion analysis devices used in the measurement of lumbar spinal mobility, Clin Biomech 14:612-619, 1999.

22. McMeeken JM, Beith ID, Newham DJ, Milligan P, Critchley DJ, The relationship between EMG and change in thickness of transversus abdominis, Clin Biomech 19:337-342, 2004.

23. Ng JK, Kippers V, Richardson CA, Muscle fibre orientation of abdominal muscles and suggested surface EMG electrode positions, Electromyogr Clin Neurophysiol 38:51-58, 1998.

24. Brown SH, McGill SM, Transmission of muscularly generated force and stiffness between layers of the rat abdominal wall, Spine 34:E70-75, 2009.

25. Marshall P, Murphy B, The validity and reliability of surface EMG to assess the neuromuscular response of the abdominal muscles to rapid limb movement, J Electromyogr Kinesiol 13:477-489, 2003.

26. Richardson CA, Hodges PW, Hides JA, Therapeutic exercises for lumbopelvic stabilization. A motor control approach for the treatment and prevention of low back pain. 2nd ed., Churchill Livingstone, London, pp. 59-73, 2004.

27. Kavcic N, Grenier S, McGill SM, Determining the stabilizing role of individual torso muscles during rehabilitation exercises, Spine 29:1254-1265, 2004.

28. Silfies SP, Squillante D, Maurer P, Wescott S, Karduna AR, Trunk muscle recruitment patterns in specific chronic low back pain populations, Clin Biomech 20:465-473, 2005. 